\title{
Evolução Clínica de Pacientes de Alto Risco Cirúrgico Submetidos a Intervenção Coronária Percutânea em Tronco da Coronária Esquerda Não-Protegido
}

\author{
Carlos Augusto Homem de Magalhães Campos ${ }^{1}$, Henrique Ribeiro ${ }^{1}$, Andre G. Spadaro ${ }^{1}$, \\ Antonio Esteves ${ }^{1}$, Marcus N. Gama ${ }^{1}$, Pedro A. Lemos ${ }^{1}$, Marco Perin ${ }^{1}$, Gilberto Marchiori ${ }^{1}$, \\ Luiz J. Kajita' ${ }^{1}$, Expedito E. Ribeiro' ${ }^{1}$, José Antonio F. Ramires ${ }^{1}$
}

\section{RESUMO}

Introdução: Recentemente, a intervenção coronária percutânea (ICP) em tronco da coronária esquerda (TCE) nãoprotegido tem se mostrado uma alternativa real à cirurgia de revascularização miocárdica. Os resultados clínicos tardios da ICP nesses pacientes são escassos na literatura, especialmente em nosso meio. Método: Registro de um centro terciário de atenção em cardiologia, que avaliou o seguimento clínico de pacientes com contraindicação à revascularização miocárdica cirúrgica submetidos a ICP em TCE, no período de 2002 a 2009. Nosso objetivo foi avaliar a mortalidade ao final de um ano. Resultados: No total, foram incluídos 77 indivíduos nesta análise, com média de idade de $65,4 \pm 13,7$ anos, dos quais 59,2\% eram do sexo masculino, $25 \%$ eram diabéticos e 3,9\% eram portadores de insuficiência renal crônica dialítica. Com relação às características angiográficas, $79,6 \%$ das lesões eram do tipo B2/C, com envolvimento do óstio em $47,4 \%$ e da bifurcação distal em $62,3 \%$. Na maioria dos casos, a indicação de ICP foi emergencial $(85,5 \%)$ e os stents farmacológicos foram utilizados em $17,5 \%$ dos pacientes. Suporte hemodinâmico por meio de balão intra-aórtico foi utilizado em $28,2 \%$ da população. Ao final de um ano a probabilidade de sobrevivência global foi de $69,7 \%$ e a quase totalidade dos óbitos ocorreu no primeiro mês de seguimento. Conclusão: Nesta população com lesão de TCE não-protegido, compreendendo pacientes de alto risco cirúrgico em situações clínicas de emergência e com grande complexidade angiográfica, a ICP é factível, com sobrevivência aceitável ao final de um ano.

DESCRITORES: Doenças cardiovasculares. Angioplastia transluminal percutânea coronária. Stents. Mortalidade.

\author{
ABSTRACT \\ Clinical Outcome in High-Surgical-Risk Patients \\ Undergoing Percutaneous Coronary Intervention \\ for Unprotected Left Main Coronary \\ Artery Disease
}

Background: Percutaneous coronary intervention ( $\mathrm{PCI}$ ) in unprotected left main coronary artery (LMCA) disease has proven to be an alternative to coronary artery bypass graft surgery $(C A B G)$. However, late clinical results of $\mathrm{PCl}$ in these patients are scarce in the literature, especially in our country. Method: Data from a tertiary cardiology center registry, which included patients with contraindications to CABG undergoing LMCA PCI, from 2002 to 2009, were analyzed. Our objective was to evaluate the 1-year mortality. Results: A total of 77 subjects were included in this analysis, mean age was $65.4 \pm 13.7$ years, $59.2 \%$ were male, $25 \%$ were diabetic, and $3.9 \%$ had renal failure requiring dialysis. Regarding the angiographic characteristics, $79.6 \%$ of lesions were type B2/C, with involvement of the ostium in $47.4 \%$ and distal bifurcation in $62.3 \%$. In most cases $\mathrm{PCl}$ was performed as an emergency procedure (85.5\%) and drug eluting stents were used in $17.5 \%$ of the patients. Hemodynamic support using an intraaortic balloon pump was used in $28.2 \%$ of the population. At the end of 1 year the probability of overall survival was $69.7 \%$ and almost all of the deaths occurred in the first month of follow-up. Conclusion: In this highly selected population with unprotected LMCA disease, including high-surgical-risk patients, in clinical emergency situations and with complex lesions, $\mathrm{PCl}$ is feasible and has acceptable 1-year mortality rates.

KEY-WORDS: Cardiovascular diseases. Angioplasty, transluminal, percutaneous coronary. Stents. Mortality.

1 Instituto do Coração do Hospital das Clínicas da Faculdade de Medicina da Universidade de São Paulo (InCor/HCFMUSP) - São Paulo, SP, Brasil.

Correspondência: Carlos Augusto Homem de Magalhães Campos. Av. Dr. Enéas Carvalho de Aguiar, 255 - Cerqueira César - São Paulo, SP, Brasil - CEP 05403-000

E-mail: cm-campos@uol.com.br

Recebido em: 11/9/2010 • Aceito em: 20/11/2010 
E mbora a cirurgia de revascularização miocárdica seja o tratamento de escolha para lesões do tronco da coronária esquerda, até recentemente as diretrizes recomendavam a intervenção coronária percutânea como alternativa de tratamento para pacientes com síndrome coronária aguda candidatos a revascularização, não elegíveis para cirurgia de revascularização miocárdica, assim como para os pacientes que requeressem intervenção de emergência por instabilidade hemodinâmica durante o cateterismo diagnóstico. ${ }^{1}$

Apesar de o tronco da coronária esquerda ser um vaso de grande calibre, a complexidade das lesões que envolvem esse território é uma das principais limitações da indicação da intervenção coronária percutânea. Cerca de dois terços das lesões acometem a bifurcação - característica anatômica associada à reestenose e à trombose do stent - e a mesma proporção desses pacientes tem doença arterial coronária multiarterial. ${ }^{2}$

Os resultados a médio e longo prazos da intervenção coronária percutânea em lesões de tronco da coronária esquerda não-protegido são escassos na literatura, especialmente em nosso meio. Este estudo foi realizado para avaliar os resultados clínicos da intervenção coronária percutânea contemporânea em pacientes com lesão de tronco da coronária esquerda não-protegido de alto risco para cirurgia de revascularização miocárdica.

\section{MÉTODO}

No período de janeiro de 2002 a dezembro de 2009, pacientes consecutivos submetidos a intervenção coronária percutânea em tronco da coronária esquerda não-protegido, com contraindicação para cirurgia de revascularização miocárdica, foram incluídos nesta análise retrospectiva, realizada em único centro brasileiro. O desfecho avaliado foi a ocorrência de óbito por qualquer causa, global e estratificado de acordo com o quadro clínico de admissão, ao final de um ano.

\section{Critérios de inclusão}

Incluímos neste estudo pacientes com lesão > 50\% em tronco da coronária esquerda não-protegido por enxertos cirúrgicos pérvios. Também incluímos pacientes com lesões em tronco da coronária esquerda nãoprotegido consequentes a injúria mecânica por procedimentos percutâneos diagnósticos ou terapêuticos. A intervenção coronária percutânea foi indicada por contraindicação à cirurgia de revascularização miocárdica no julgamento do médico assistente do paciente, seguindo as diretrizes vigentes e levando-se em conta a necessidade de revascularização imediata em situações de urgência, comorbidades associadas a coronariopatia e fragilidade física do paciente.

\section{Definições}

O diagnóstico de infarto agudo do miocárdio com supradesnivelamento do segmento ST foi realizado na ocorrência de dor típica em repouso associada a elevação persistente de ST maior que $1 \mathrm{~mm}$ em duas derivações contíguas ou novo bloqueio de ramo esquerdo ao eletrocardiograma. Síndrome coronária aguda sem supradesnivelamento do segmento ST foi definida como dor em repouso, sem os critérios eletrocardiográficos citados anteriormente, associada ou não a elevação dos marcadores de necrose miocárdica. Angina estável foi considerada na presença de dor típica desencadeada por esforço, isquemia silenciosa documentada por prova funcional ou equivalente anginoso. A morfologia das lesões foi classificada de acordo com as definições do American College of Cardiology/American Heart Association (ACC/AHA) modificadas por Ellis et al. ${ }^{3}$.

\section{Coleta e análise de dados}

Os dados da evolução intra-hospitalar foram coletados por médicos treinados, durante a internação índice, seguindo o preenchimento de formulários previamente padronizados. A coleta incluiu características clínicas, resultados de exames laboratoriais, dados do procedimento invasivo e evolução clínica até a alta hospitalar. A coleta de informações na evolução tardia foi realizada por seguimento ambulatorial, revisão dos registros hospitalares ou contato telefônico.

As variáveis contínuas foram descritas como média \pm desvio padrão e comparadas por ANOVA. As variáveis categóricas foram descritas como porcentagem e comparadas com o teste de qui-quadrado. As análises foram realizadas com programa estatístico SPSS 13.0, e um valor de $\mathrm{P}<0,05$ foi considerado estatisticamente significante para todos os testes.

\section{RESULTADOS}

Entre 2002 e 2009, 77 pacientes foram submetidos a intervenção coronária percutânea em tronco da coronária esquerda não-protegido em nossa instituição. Conforme demonstrado na Tabela 1, a média de idade foi de $65,4 \pm 13,7$ anos, $59,2 \%$ eram do sexo masculino e $25 \%$, diabéticos. Na maioria dos casos $(85,5 \%)$ as intervenções coronárias percutâneas foram realizadas em situações agudas, sendo 56,6\% em síndromes coronárias agudas e 28,9\% por complicações de procedimentos percutâneos. Algumas comorbidades associadas atestam a gravidade desse grupo, como insuficiência cardíaca em cerca de um quarto dos casos e insuficiência renal crônica dialítica em quase $5 \%$ dos pacientes. Na comparação dos subgrupos, apesar de encontrarmos grande variabilidade na prevalência de algumas características clínicas, as diferenças não tiveram significância estatística.

As variáveis angiográficas e do procedimento estão listadas na Tabela 2. Lesões do tipo B2/C foram encontradas em $79,6 \%$ dos casos. Houve envolvimento do óstio da coronária esquerda em metade das in- 
TABELA 1

Características clínicas

\begin{tabular}{lcccccc}
\hline & $\begin{array}{c}\text { Global } \\
(\mathbf{n}=\mathbf{7 7})\end{array}$ & $\begin{array}{c}\text { IAMCSST } \\
(\mathbf{n}=\mathbf{2 0})\end{array}$ & $\begin{array}{c}\text { SCASSST } \\
(\mathbf{n}=\mathbf{2 4})\end{array}$ & $\begin{array}{c}\text { Angina estável } \\
(\mathbf{n}=\mathbf{1 1})\end{array}$ & $\begin{array}{c}\text { latrogênico } \\
(\mathbf{n}=\mathbf{2 2})\end{array}$ & $\mathbf{P}$ \\
\hline Idade, anos & $65,4 \pm 13,7$ & $61,5 \pm 16,5$ & $70,8 \pm 13,4$ & $61,8 \pm 9,3$ & $65 \pm 11,7$ & 0,1 \\
Sexo masculino, \% & 59,2 & 40 & 59,1 & 81,8 & 68,2 & 0,1 \\
HAS, \% & 82,9 & 75 & 82,6 & 81,8 & 90,9 & 0,59 \\
Diabetes, \% & 25 & 20 & 39,1 & 18,2 & 18,2 & 0,31 \\
Dislipidemia, \% & 53,9 & 50 & 56,5 & 45,5 & 59,1 & 0,86 \\
Tabagismo, \% & 31,6 & 35 & 21,7 & 36,4 & 36,4 & 0,68 \\
IC, \% & 23,7 & 15 & 34,8 & 9,1 & 27,3 & 0,27 \\
IRC dialítica, \% & 3,9 & 5 & 8,7 & 0 & 0 & 0,42 \\
IAM prévio, \% & 19,7 & 15 & 30,4 & 9,1 & 18,2 & 0,42 \\
AVC prévio, \% & 2,6 & 5 & 0 & 0 & 4,5 & 0,64 \\
\hline
\end{tabular}

AVC = acidente vascular cerebral; HAS = hipertensão arterial sistêmica; IAM = infarto agudo do miocárdio; IAMCSST = infarto agudo do miocárdio com supradesnivelamento do segmento ST; IC = insuficiência cardíaca; IRC = insuficiência renal crônica; $\mathrm{n}=$ número de pacientes; SCASSST = síndrome coronária aguda sem supradesnivelamento do segmento ST.

tervenções $(47,4 \%)$, e comprometimento da bifurcação do tronco da coronária esquerda em quase dois terços dos casos $(62,3 \%)$. No total, os stents farmacológicos foram utlizados em $17,5 \%$ dos pacientes, variando de $6,7 \%$ no infarto agudo do miocárdio com supradesnivelamento do segmento ST a 33,3\% nas síndromes coronárias agudas sem supradesnivelamento do segmento ST. Suporte hemodinâmico por meio de balão intra-aórtico foi utilizado em $28,2 \%$ da população. Novamente, na análise dos subgrupos, encontramos variabilidade na prevalência de algumas características angiográficas e do procedimento, mas as comparações não demonstraram diferenças estatisticamente significantes.

Ao final de um ano de acompanhamento, a sobrevivência estimada por meio da curva de Kaplan-Meier foi de $69,7 \%$ e a quase totalidade dos óbitos ocorreu no primeiro mês de seguimento (Figura 1). Quando comparamos a mortalidade em um ano, de acordo com os quadros clínicos de apresentação, observamos que houve variação de 18,2\% no grupo iatrogênico a 40\% no grupo de infarto agudo do miocárdio com supradesnivelamento do segmento ST, com cifras intermediárias nos grupos de síndrome coronária aguda sem supradesnivelamento do segmento ST $(30,4 \%)$ e de angina estável $(36,4 \%, P=0,45)$.

\section{DISCUSSÃO}

Este registro unicêntrico brasileiro demonstrou que a probabilidade de sobrevivência em um ano de pacientes submetidos a intervenção coronária percutânea em tronco da coronária esquerda não-protegido, não candidatos a cirurgia de revascularização miocárdica, é de $70 \%$. Podemos considerar essa probabilidade de sobrevivência aceitável, pela elevada complexidade tanto clínica como angiográfica dos indivíduos trata- dos. A presença de estenose significativa no tronco da coronária esquerda está associada a elevado risco de ocorrência de desfechos adversos significativos, como morte súbita e infarto agudo do miocárdio fatal, relacionado à grande quantidade de miocárdio em risco. ${ }^{1}$

Colocando esses dados em perspectiva, registro de Hurtado et al. ${ }^{4}$, que avaliou 71 pacientes com intervenção coronária percutânea emergencial em tronco da coronária esquerda, encontrou mortalidade de $47 \%$ no período hospitalar. De forma semelhante, Dubois et al. ${ }^{5}$ encontraram mortalidade de $22 \%$ ao final de um ano em 143 pacientes submetidos a tratamento percutâneo em tronco da coronária esquerda na presença de perfil de risco elevado. ${ }^{5}$ Nos casos de injúria iatrogênica ao tronco da coronária esquerda, a mortalidade pode chegar a $100 \%$, de acordo com o grau de comprometimento do tronco da coronária esquerda pela dissecção. ${ }^{6}$ No entanto, a presente análise é ainda mais específica, pois trata exclusivamente de pacientes com contraindicação formal à cirurgia, seja pela imediata necessidade de revascularização seja pelo estado clínico geral dos indivíduos.

Na indicação de intervenção coronária percutânea, deve-se ressaltar que a maioria $(85,5 \%)$ dos pacientes foi tratada em situação de urgência, por apresentar síndrome coronária aguda e instável $(56,6 \%)$ ou complicações intervencionistas do procedimento percutâneo com necessidade de correção imediata (28,9\%). Estudos recentes têm demonstrado uma tendência mundial ao incremento das indicações das intervenções coronárias percutâneas em tronco da coronária esquerda em síndromes coronárias agudas. Nesse sentido, o Global Registry of Acute Coronary Events (registro GRACE) incluiu 43.018 pacientes com síndromes coronárias agudas de 14 países das Américas do Norte 
TABELA 2

Características angiográficas e do procedimento

\begin{tabular}{|c|c|c|c|c|c|c|}
\hline & $\begin{array}{l}\text { Global } \\
(\mathbf{n}=77)\end{array}$ & $\begin{array}{l}\text { IAMCSST } \\
(\mathbf{n}=\mathbf{2 0})\end{array}$ & $\begin{array}{r}\text { SCASSST } \\
(\mathbf{n}=24)\end{array}$ & $\begin{array}{l}\text { Angina estável } \\
\qquad(\mathrm{n}=11)\end{array}$ & $\begin{array}{l}\text { Iatrogênico } \\
(\mathrm{n}=22)\end{array}$ & $\mathbf{P}$ \\
\hline Diâmetro do stent, mm & $3,5 \pm 0,5$ & $3,5 \pm 0,6$ & $3,4 \pm 0,6$ & $3,5 \pm 0,4$ & $3,6 \pm 0,4$ & 0,71 \\
\hline Comprimento do stent, $\mathrm{mm}$ & $16,3 \pm 6$ & $16,3 \pm 5,7$ & $17 \pm 6,1$ & $14,6 \pm 7,6$ & $16 \pm 5,8$ & 0,82 \\
\hline Lesões de bifurcação, \% & 62,3 & 47,4 & 73,9 & 54,5 & 50 & 0,8 \\
\hline Lesão ostial, \% & 47,4 & 50 & 39,1 & 54,5 & 50 & 0,8 \\
\hline Stent farmacológico, \% & 17,5 & 6,7 & 33,3 & 14,3 & 7,1 & 0,1 \\
\hline Técnica de bifurcação & & & & & & 0,8 \\
\hline 1 stent & 44,2 & 55 & 58,3 & 63,6 & 81,2 & \\
\hline 2 stents & 55,8 & 45 & 41,7 & 36,4 & 18,2 & \\
\hline Emprego de BIA, \% & 28,2 & 38,9 & 26,1 & 33,3 & 19 & 0,56 \\
\hline
\end{tabular}

BIA = balão intra-aórtico; IAMCSST = infarto agudo do miocárdio com supradesnivelamento do segmento ST; $\mathrm{n}=$ número de pacientes; SCASSST = síndrome coronária aguda sem supradesnivelamento do segmento ST.

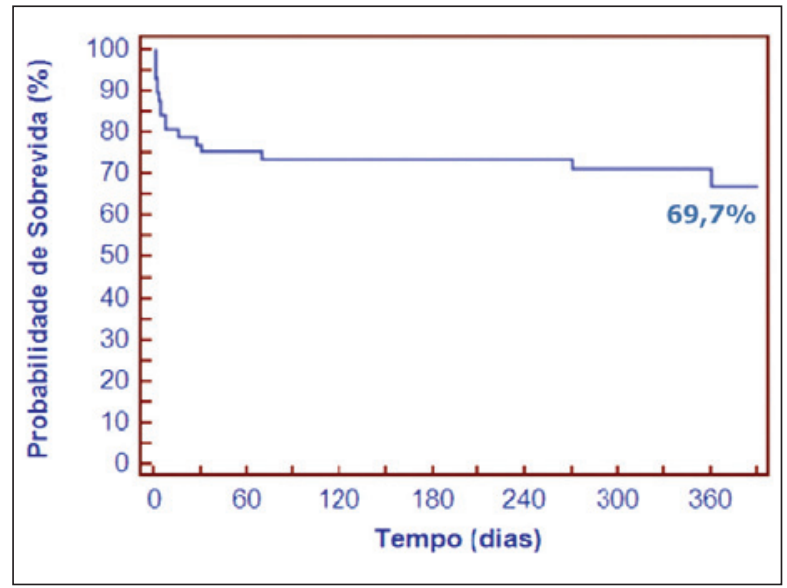

Figura 1 - Probabilidade de sobrevivência ao final de um ano de seguimento.

e Sul, da Europa, da Austrália e da Nova Zelândia. Desse total, 1.799 pacientes (4\%) tinham lesão significativa em tronco da coronária esquerda e, nessa população, a intervenção coronária percutânea demonstrou aumento significativo de sua indicação ao longo dos anos. Também pode ser observado que o escore de risco GRACE foi significativamente mais elevado no grupo de tratamento percutâneo vs. cirurgia de revascularização miocárdica, refletindo a seleção de pacientes para a revascularização cirúrgica. Após seis meses de seguimento, a mortalidade foi significativamente reduzida com os métodos de revascularização miocárdica vs. tratamento clínico [hazard ratio (HR) 0,45, intervalo de confiança de 95\% (IC 95\%) 0,23-0,85 para intervenção coronária percutânea; HR 0,11, IC 95\% 0,040,28 para cirurgia de revascularização miocárdica]. ${ }^{7}$ De forma análoga, outro estudo multicêntrico prospectivo comparou a intervenção coronária percutânea $(n=63)$ com a cirurgia de revascularização miocárdica $(\mathrm{n}=75)$ nas síndromes coronárias agudas, mostrando tendência para menor mortalidade aos 30 dias com o tratamento percutâneo vs. cirúrgico $(P=0,07){ }^{8}$

Além do diagnóstico de admissão, várias características clínicas e angiográficas são marcadores de gravidade desta população. Quase um quarto dos indivíduos apresentava insuficiência cardíaca e proporção semelhante era de diabéticos. Quase 5\% tinham doença renal avançada e 2,6\% tinham acidente vascular cerebral prévio. Todas essas características clínicas estão associadas a pior prognóstico, acometimento coronário mais difuso ou pior status vital. ${ }^{9-13}$ As características angiográficas também refletem complexidade desse grupo, já que quase dois terços das lesões envolviam a bifurcação distal do tronco e quase metade apresentava acometimento do segmento aorto-ostial. Lesões complexas do tipo B2/C (ACC/AHA) constituíram praticamente $80 \%$ das intervenções. Essas características dificultam a realização técnica da intervenção e, dessa forma, influenciam diretamente os resultados. ${ }^{3,14}$

Apesar de os stents farmacológicos sabidamente produzirem redução da necessidade de revascularização ou até da mortalidade nas intervenções coronárias percutâneas em tronco da coronária esquerda não-protegido ${ }^{15,16}$, essas próteses foram utilizadas em somente $17,5 \%$ dos casos. Esse dado pode ser explicado pelo fato de no Brasil, atualmente, a utilização de stents farmacológicos ser majoritária em pacientes atendidos na Saúde Complementar, como também pelo fato de o sistema público de saúde não dispor dessa tecnologia. ${ }^{17}$ A realização de intervenções em caráter de emergência dificulta ainda mais o emprego dos stents farmacológicos. Como limitações, os autores reconhecem que esse é um estudo retrospectivo, unicêntrico, com número pequeno de pacientes. Variáveis que podem influenciar a evolução, como função ventricular 
esquerda, e dados de angiografia coronária quantitativa, que avaliam os resultados do procedimento, não puderam ser obtidos. No entanto, esta é primeira publicação nacional que trata dessa população de grande complexidade tanto clínica como angiográfica, preenchendo a lacuna de conhecimento relativa à evolução a médio prazo de pacientes submetidos a intervenção coronária percutânea em tronco da coronária esquerda não-protegido, de alto risco cirúrgico.

\section{CONCLUSÃO}

Pacientes submetidos a intervenção coronária percutânea em tronco da coronária esquerda não-protegido, de alto risco cirúrgico, em situações clínicas de emergência e com grande complexidade angiográfica demonstraram que o procedimento é factível, com taxa de sobrevida em um ano compatível com a gravidade clínica dos indivíduos.

\section{CONFLITO DE INTERESSES}

Os autores declararam inexistência de conflito de interesses relacionado a este manuscrito.

\section{REFERÊNCIAS}

1. King SB $3^{\text {rd }}$, Smith SC Jr, Hirshfeld JW Jr, Jacobs AK, Morrison DA, Williams DO, et al. 2007 Focused Update of the ACC/ AHA/SCAI 2005 Guideline Update for Percutaneous Coronary Intervention: a report of the American College of Cardiology/ American Heart Association Task Force on Practice Guidelines: 2007 Writing Group to Review New Evidence and Update the ACC/AHA/SCAI 2005 Guideline Update for Percutaneous Coronary Intervention, Writing on Behalf of the 2005 Writing Committee. Circulation. 2008;117(2):261-95.

2. European Association for Percutaneous Cardiovascular Interventions; Wijns W, Kolh P, Danchin N, DI MC, Falk V, Folliguet $\mathrm{T}$, et al. Guidelines on myocardial revascularization. Task Force on Myocardial Revascularization of the European Society of Cardiology (ESC) and the European Association for Cardio-Thoracic Surgery (EACTS). Eur Heart J. 2010;31(20): 2501-55.

3. Ellis SG, Vandormael MG, Cowley MJ, DiSciascio G, Deligonul U, Topol EJ, et al. Coronary morphologic and clinical determinants of procedural outcome with angioplasty for multivessel coronary disease: implications for patient selection. Multivessel Angioplasty Prognosis Study Group. Circulation. 1990;82(4):1193-202.

4. Hurtado J, Pinar Bermúdez E, Redondo B, Lacunza Ruiz J, Gimeno Blanes JR, García de Lara J, et al. Emergency percutaneous coronary intervention in unprotected left main coronary arteries. Predictors of mortality and impact of cardiogenic shock. Rev Esp Cardiol. 2009;62(10):1118-24.

5. Dubois C, Dens J, Sinnaeve P, Belmans A, Van Cleemput J,
Mendez M, et al. Results of percutaneous coronary intervention of the unprotected left main coronary artery in 143 patients and comparison of 30-day mortality to results of coronary artery bypass grafting. Am J Cardiol. 2008;101(1):75-81.

6. Eshtehardi $\mathrm{P}$, Adorjan $\mathrm{P}$, Togni $\mathrm{M}$, Tevaearai $\mathrm{H}$, Vogel $\mathrm{R}$, Seiler $\mathrm{C}$, et al. latrogenic left main coronary artery dissection: incidence, classification, management, and long-term followup. Am Heart J. 2010;159(6):1147-53.

7. Montalescot G, Brieger D, Eagle KA, Anderson FA Jr, FitzGerald G, Lee MS, et al.; GRACE Investigators. Unprotected left main revascularization in patients with acute coronary syndromes. Eur Heart J. 2009;30(19):2308-17.

8. Buszman PP, Bochenek A, Konkolewska M, Trela B, Kiesz RS, Wilczyñski M, et al. Early and long-term outcomes after surgical and percutaneous myocardial revascularization in patients with non-ST-elevation acute coronary syndromes and unprotected left main disease. J Invasive Cardiol. 2009;21(11):564-9.

9. Singh M, Holmes DR Jr, Lennon RJ, Rihal CS. Development and validation of risk adjustment models for long-term mortality and myocardial infarction following percutaneous coronary interventions. Circ Cardiovascular Interv. 2010;3(5):423-30.

10. Singh M, Lennon RJ, Holmes DR Jr, Bell MR, Rihal CS. Correlates of procedural complications and a simple integer risk score for percutaneous coronary intervention. J Am Coll Cardiol. 2002;40(3):387-93.

11. Singh M, Rihal CS, Selzer F, Kip KE, Detre K, Holmes DR. Validation of Mayo Clinic risk adjustment model for inhospital complications after percutaneous coronary interventions, using the National Heart, Lung, and Blood Institute dynamic registry. J Am Coll Cardiol. 2003;42(10):1722-8.

12. Moscucci M, Kline-Rogers E, Share D, O'Donnell M, MaxwellEward A, Meengs WL, et al. Simple bedside additive tool for prediction of in-hospital mortality after percutaneous coronary interventions. Circulation. 2001;104(3):263-8.

13. Min SY, Park DW, Yun SC, Kim YH, Lee JY, Kang SJ, et al. Major predictors of long-term clinical outcomes after coronary revascularization in patients with unprotected left main coronary disease: analysis from the MAIN-COMPARE Study. Circ Cardiovasc Interv. 2010;3(2):127-33.

14. Williams DO, Holubkov R, Yeh W, Bourassa MG, AlBassam M, Block PC, et al. Percutaneous coronary intervention in the current era compared with 1985-1986: the National Heart, Lung, and Blood Institute Registries. Circulation. 2000; 102(24):2945-51.

15. Kim YH, Park DW, Lee SW, Yun SC, Lee CW, Hong MK, et al. Long-term safety and effectiveness of unprotected left main coronary stenting with drug-eluting stents compared with bare-metal stents. Circulation. 2009;120(5):400-7.

16. Pandya SB, Kim YH, Meyers SN, Davidson CJ, Flaherty JD, Park DW, et al. Drug-eluting versus bare-metal stents in unprotected left main coronary artery stenosis - a meta-analysis. JACC Cardiovasc Interv. 2010;3(6):602-11.

17. Campos CAHM, Quadros AS, Welter DI, Camozzatto F, Rovinski D, Sarmento-Leite R, et al. Associação entre renda, características clínicas e angiográficas de pacientes submetidos a intervenção coronária percutânea. Rev Bras Cardiol Invasiva. 2010;18(1):44-9. 